\title{
El Aprendizaje Basado en Problemas como estrategia didáctica en el proceso de enseñanza
}

\section{Problem-Based Learning as a didactic strategy in the teaching process}

\author{
Norma Isabel Palta Valladares ${ }^{1}$, Juan Patricio Sigüenza Orellana ${ }^{1}$ y Jhoana Fernanda Pulla Merchán ${ }^{1}$ \\ ${ }^{1}$ Universidad Católica de Cuenca \\ *Inpalta@ucacue.edu.ec
}

DOI: https://doi.org/10.26871/killkana_social.v2i2.291

\begin{abstract}
Resumen
El presente artículo pretende determinar la utilización del Aprendizaje Basado en Problemas como estrategia didáctica en el proceso de enseñanza, para ello se identificó el nivel de conocimientos y el estado actual de utilización del ABP en el proceso de enseñanza aprendizaje. El trabajo de campo se realizó en la Escuela de Educación General Básica José de la Vega del cantón Paute, provincia del Azuay, Ecuador en el año académico 2016-2017. Se aplicaron encuestas a docentes y estudiantes del octavo, noveno y décimo año de Educación General Básica; además se realizó un análisis de las Planificaciones Didácticas, empleándose para ello una matriz de revisión documental para cada documento. Se identificó que el $\mathrm{ABP}$, es una estrategia didáctica que permite que el educando construya su aprendizaje mediante la participación activa, investigativa y crítica llevándole a un aprendizaje significativo, además se comprobó que el nivel de conocimientos sobre el ABP es casi nulo en docentes y estudiantes, así como su utilización en los procesos de enseñanza.
\end{abstract}

Palabras clave: Aprendizaje Basado en Problemas, Ciclo del Aprendizaje, Metodología de Enseñanza, Aprendizaje.

\begin{abstract}
The purpose was to determine the use of Problem Based Learning (PBL) as a didactic strategy in the teaching process. Fieldwork was carried out at the José de la Vega Basic General Education School in Paute, province of Azuay, Ecuador, in the academic year 2016-2017. With teachers, male and female students from the eighth, ninth and tenth of General Basic Education. The survey technique was used by using printed forms; in addition, an analysis of the Didactic Schedules was made using a document review matrix for each document. Identifying that PBL is a didactic strategy that allows the learner to construct their learning through active, investigative and critical participation leading to a significant learning; in addition, it was identified that the level of knowledge about the PBL is almost null in the teachers, as well as its use in teaching process.
\end{abstract}

Key words: Problem-Based Learning, Learning Cycle, Teaching Methodology, Learning.

\section{Introducción}

La estrategia del Aprendizaje Basado en Problemas (ABP) no es nueva en el contexto educativo. Sus orígenes están aproximadamente en los años 60 en la Universidad MacMaster de Canadá en la Facultad de Medicina, pues esta metodología nace con el firme propósito de mejorar la formación de profesionales en esa área, ya que los estudiantes se formaban con las estrategias didácticas del método tradicional el cual se basa en clases magistrales y un dominio predominante del contenido teórico(Arpí Miró y cols., 2012).

Por lo tanto, es necesario abordar las estrategias didácticas basadas en el método tradicional y reemplazarlas por estrategias innovadoras, las mismas que se centran en la actividad del educando para la construcción de su conocimiento. En tal virtud, la presente investigación busca determinar si en el contexto educativo ecuatoriano se utiliza el Aprendizaje Basado en Problemas como estrategia didáctica en el proceso de enseñanza, ya que se la concibe como una estrategia que requiere que los educandos se involucren de forma activa en su propio aprendizaje para desarrollar la formación auto dirigida. Es decir, el docente deja de ser el protagonista del proceso de enseñanza para convertirse en un facilitador, guía, asesor o tutor y exige que el protagonista del proceso de enseñanza-aprendizaje sea el educando a través de una participación activa en 
la construcción del conocimiento(Escribano y Del Valle, 2008).

Por ello Barrows (1986) manifiesta que todo docente debe conocer las características del ABP, las cuales se describen a continuación:

- $\quad$ El aprendizaje está centrado en el estudiante.

- El aprendizaje se aprende en pequeños grupos.

- Los profesores son facilitadores o guías de este proceso.

- Los problemas son el foco de organización y el estímulo para el aprendizaje.

- Los problemas son un vehículo para el desarrollo de habilidades en la resolución de problemas.

- La nueva información se adquiere a través del aprendizaje auto dirigido.

Además de estas características, el ABP promueve la participación activa de los estudiantes con el fin de que sean capaces y responsables de proponer la solución a un problema planteado, solución que debe surgir del trabajo en equipo. Esto evidencia el trabajo colaborativo y cooperativo y a su vez promueve el desarrollo de otras competencias a lo largo de su formación.

Por otro lado, es necesario conocer las ventajas que implica la aplicación de la estrategia didáctica del ABP, tal como lo reconoce la Dirección de Invetigación y Desarrollo Educativo - Vicerrectoría Académica del Instituto Tecnológico y de Estudios Superiores de Monterrey(2008), quienes destacan que el ABP promueve:

- Estudiantes con mayor motivación.

- Un aprendizaje más significativo.

- Desarrollo de habilidades de pensamiento.

- Desarrollo de habilidades para el aprendizaje e integración de un modelo de trabajo.

- Posibilita mayor retención de información.

- Permite la integración del conocimiento.

Teniendo claras las características y ventajas del ABP, es menester describir el rol del docente en la aplicación de esta estrategia, rol que requiere que el docente deje de ser el centro del proceso de enseñanza-aprendizaje para convertirse en tutor, asesor $\mathrm{u}$ orientador ante las inquietudes de sus estudiantes. A continuación se mencionan algunas funciones del docente en el desarrollo de esta metodología:

- Da un papel protagonista al estudiante en la construcción de su aprendizaje.

- Tiene que ser consciente de los logros que consiguen sus estudiantes.

- Es un guía, tutor, un facilitador del aprendizaje que acude a los estudiantes cuando le necesitan y les ofrece información cuando es preciso.

- El papel principal es ofrecer a los estudiantes diversas oportunidades de aprendizaje.

- Ayuda a sus estudiantes a pensar críticamente, orientando sus reflexiones y formulando cuestionamientos importantes.

- Realizar sesiones de tutoría con los estudiantes (De innovación educativa, 2008, p.12).
Como se describe, el papel del docente en el ABP es de guía, el mismo que orienta, asesora o sugiere mecanismos para superar las inquietudes que se presentan en la resolución del problema planteado, pero siempre dejando la responsabilidad de aprendizaje a los mismos educandos.

En cambio el rol del estudiante en esta estrategia es de un sujeto activo que aprende por medios propios y sugeridos, dejando atrás su pasividad en el proceso de enseñanza, para lo cual debe desarrollar ciertas características:

- Asumir su responsabilidad ante el aprendizaje.

- Trabajar con diferentes grupos gestionando los posibles conflictos que surjan.

- Disponer de las estrategias necesarias para planificar, controlar y evaluar los pasos que lleva a cabo en su aprendizaje.

El estudiante tiende a ser un sujeto con responsabilidades propias de las cuales dependerá su aprendizaje, además debe tener presente que para la resolución del problema planteado cuenta con un equipo de trabajo en donde cada sujeto colabora en la resolución del problema.

Teniendo claras las funciones del docente y educando, es necesario describir el proceso de planificación del ABP, el mismo que inicia con el diseño de un problema real, dicho diseño está a cargo del docente. Para Morales y Landa(2004), el problema debe plantear un conflicto cognitivo, debe ser retador, interesante y motivador para que el estudiante se interese por buscar la solución al problema planteado.

En la planificación del problema se deben tener presentes los siguientes aspectos:

- Seleccionar los objetivos que los estudiantes buscarán alcanzar.

- Escoger la situación problema, la misma que debe ser lo suficientemente compleja, pero no imposible de resolver.

- Orientar las reglas de la actividad y el trabajo en equipo para cuando existan conflictos entre compañeros.

- Establecer un tiempo y especificarlo para la resolución del problema, y se debe tomar en cuenta que el tiempo no sea demasiado largo.

- Organizar sesiones de tutorías para atender inquietudes (De innovación educativa, 2008, p.7).

Una vez que se ha diseñado el problema se debe plantear para que sea resuelto, y se propone el siguiente esquema metodológico del ABP, el mismo que contiene ocho pasos que se describen a continuación:

1) Leer y analizar el escenario del problema.

2) Realizar una lluvia de ideas.

3) Hacer una lista de aquello que se conoce.

4) Hacer una lista de aquello que se desconoce.

5) Hacer una lista de aquello que se necesita para resolver el problema.

6) Definir el problema.

7) Obtener información.

8) Presentar resultados (Morales Bueno y Landa Fitzgerald, 2004, p.153). 
El esquema propuesto puede emplearse en varias sesiones de clases, pues la resolución de un problema no se realiza en una sola hora de clase.

Para concluir con éxito la aplicación del ABP, los docentes deben considerar que esta estrategia requiere la aplicación de los componentes de autoevaluación, coevaluación y heteroevaluación. Es decir, los estudiantes deben tener la posibilidad de evaluarse a sí mismos, a sus compañeros y al tutor. Entre los criterios a ser evaluados se debe considerar el aporte de cada miembro al grupo de trabajo, las asesorías del docente y los resultados obtenidos (PootDelgado, 2013).

La evaluación del ABP también requiere un cambio en la forma de evaluar, pues si bien es cierto que se emplean los mismos instrumentos de la evaluación convencional, el cambio está en la forma en que se emplean dichos instrumentos, pues no se pueden evaluar aspectos que reflejen memorización del contenido sino por el contrario, que evidencien el análisis, reflexión y crítica en la construcción del conocimiento. Monterrey (2008), menciona algunos instrumentos de evaluación que pueden emplearse en la evaluación del ABP:

a) Examen escrito,

b) Examen práctico,

c) Mapas conceptuales,

d) Evaluación del compañero, autoevaluación, evaluación al tutor,

e) Presentación oral y

f) Reporte escrito.

Realizada la descripción de los componentes teóricos y prácticos, tales como concepciones generales, procesos de aplicabilidad y sistemas de evaluación, se puede establecer que el presente trabajo de investigación es diferente de otros porque no existe un estudio del ABP en el área de Educación General Básica (EGB), sin embargo se identifica el trabajo de Palta (2016) aplicado en la Universidad Católica de Cuenca. Dicho trabajo se realizó para identificar la aplicación del ABP en el ámbito universitario y posteriormente proponer la estructura didáctica para su correcta implementación en el proceso de enseñanza. Otros estudios se identifican en la Universidad Casa Grande de Guayaquil, la misma presentó el ABP como un enfoque pedagógico en estudiantes de postgrado de Neonatología, con la implementación y la aplicación del ABP para que los estudiantes puedan enfrentarse a un problema incierto como lo hacen los expertos en la vida real (Chávez, 2009).

Como se describe anteriormente, las investigaciones están encaminadas a las áreas universitarias, en cambio el presente estudio se centra en el área de EGB, ya que en este ámbito el proceso de enseñanza-aprendizaje se diferencia del área universitaria. En la Educación General Básica, la propuesta a trabajar con la metodología del ABP, debe articularse al logro de los resultados de aprendizaje, los mismos que están declarados como una política pública en el ámbito de la educación ecuatoriana.

\section{Metodología}

El presente trabajo de investigación tiene un enfoque cuali-cuantitativo, el mismo que permitió medir y obtener resultados en cuanto al nivel de conocimiento de los docentes sobre el ABP y el estado de utilización del ABP en básica superior de la Escuela de Educación General Básica José de la Vega del cantón Paute, provincia del Azuay. La investigación cualitativa, como indica su propia denominación, tiene como objetivo la descripción de las cualidades de un fenómeno. Busca un concepto que pueda abarcar una parte de la realidad. No se trata de probar o de medir en qué grado una cualidad se encuentra en un cierto acontecimiento dado, sino de descubrir tantas cualidades como sea posible y la investigación cuantitativa es la que analiza diversos elementos que pueden ser medidos y cuantificados. Toda la información se obtiene a base de muestras de la población, y sus resultados son extrapolables a toda la población, con un determinado nivel de error y nivel de confianza (Palacios, 2006).

Para la recolección de información se desarrolló una investigación de tipo exploratoria y descriptiva. Esta investigación es exploratoria, porque busca una adecuada implementación del ABP en el proceso de enseñanza y descriptiva porque se especifican características, ventajas, desventajas, propósito o situaciones del fenómeno que se estudia.

La población en estudio corresponde a 5 docentes, 66 estudiantes de básica superior y 106 documentos de planificación curricular. Al no cumplir con los requisitos para la selección de la muestra de docentes y estudiantes, se consideró el total de la población.

Para la obtención de la muestra en los documentos curriculares se trabajó con un margen de error del $5 \%, 50 \%$ de heterogeneidad y $95 \%$ de confiabilidad, dando como resultado 84 documentos a ser analizados.

El procedimiento para el desarrollo de la presente investigación se inició con la elaboración de los cuestionarios, luego se solicitó la valoración a dos expertos en el área académica, catedráticos de la Universidad Católica de Cuenca, los mismos que observaron que los ítems propuestos responden a contribuir el alcance de los objetivos planteados; sin embargo, se hicieron observaciones en cuanto a la forma de diagramación de los instrumentos; así como ciertos términos en algunas preguntas planteadas.

Los cuestionarios estuvieron diseñados de acuerdo a las dos variables planteadas: Aprendizaje Basado en Problemas y la estrategia didáctica, esta última es la instancia que acoge a los métodos, técnicas y procedimientos didácticos; que permitirán al docente dirigir con pericia el aprendizaje de sus estudiantes (Carrasco, 2004).

Para el análisis de datos se realizaron los cuadros estadísticos de todas las variables objeto de estudio, obteniendo de manera ágil los resultados, lo que permitió identificar omisiones en las diferentes preguntas, posteriormente se representó la información en tablas, las mismas que fueron dispuestas en función a los ámbitos de investigación. 
Al tener la investigación un enfoque cuali-cuantitativo se reconoce los niveles de conocimiento, así como el estado de utilización del ABP, información que es contextualizada según el entorno en el que se presentó el problema.

En la siguiente tabla se describen las técnicas e instrumentos para la recolección de información:

Tabla 1 Técnicas e instrumentos para la recolección de información

\begin{tabular}{cccc}
\hline $\begin{array}{c}\text { Tipo de } \\
\text { instrumento }\end{array}$ & Informante & $\begin{array}{c}\text { Instrumento } \\
\text { de recolección }\end{array}$ & $\begin{array}{c}\text { Instrumento } \\
\text { de registro }\end{array}$ \\
\hline $\begin{array}{c}\text { Encuesta } \\
\text { Matriz de revisión } \\
\text { documental del } \\
\begin{array}{c}\text { Plan de Unidad } \\
\text { Didáctica }\end{array}\end{array}$ & $\begin{array}{c}\text { Docentes } \\
\text { académicos }\end{array}$ & Cuestionario & Encuesta \\
Encuesta & Estudiantes & Cuestionario & Matriz \\
\hline
\end{tabular}

Elaborado: Autores, 2017

\section{Análisis de resultados}

Se procedió con un análisis descriptivo y correlacional de todas las variables.

Tabla 2 Usted tiene conocimiento del proceso didáctico para la aplicación del $A B P$

\begin{tabular}{lll}
\hline & Fr. & Por. \\
\hline Totalmente & 2 & $40 \%$ \\
En su mayor parte & 3 & $60 \%$ \\
Parcialmente & 0 & $0 \%$ \\
Ninguno & 0 & $0 \%$ \\
Total & 5 & $100 \%$ \\
\hline
\end{tabular}

Fuente: Encuesta a docentes

Elaborado: Autores, 2017

Analizada la tabla 2 que corresponde a la dimensión de aplicabilidad del ABP se identifica que el $40 \%$ de los docentes reconocen tener un conocimiento total del proceso didáctico para la aplicación del $\mathrm{ABP}$, lo cual resulta contradictorio ya que en la tabla 4 el $50 \%$ de los estudiantes manifiestan que los trabajos en grupo son para exposiciones de temas abordados en clase, aspecto que de acuerdo a la fundamentación conceptual es erróneo ya que los trabajos en grupo son para debatir, plantear, investigar, etc. Además en la tabla 6 el 83, $3 \%$ de los estudiantes reconocen que a veces los docentes aplican tutorías para el desarrollo de los trabajos, aspecto que de acuerdo a la fundamentación conceptual debería ser predominante en las actividades de enseñanza. Por otro lado en la matriz de revisión documental se evidencia un desconocimiento de los docentes en el sistema integral de evaluación del ABP ya que el $100 \%$ de los docentes aplica solo la evaluación sumativa.
Tabla 3 Aplicabilidad del $A B P$

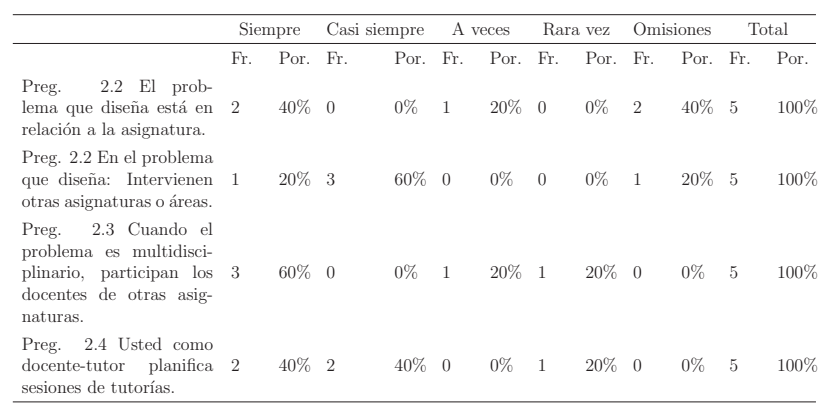

Fuente: Encuesta a estudiantes

Elaborado: Autores 2017

Analizada la tabla 3 que corresponde a la dimensión de aplicabilidad del ABP se identifica que el $60 \%$ de los docentes reconoce que simpre en el diseño de un problema intervienen docentes de otras asignaturas, lo cual resulta contradictorio ya que en la revisión documental de las planificaciones se evidencia que el $100 \%$ de los docentes aplica la estrategia del ciclo del aprendizaje y no el ABP. Por otro lado, los docentes no reconocen la importancia del trabajo en equipo y la tutoría que deben brindar los docentes, elementos que según la fundamentación conceptual son esenciales para el éxito del ABP.

En base al presente análisis el nivel de conocimientos que los docentes disponen sobre la aplicabilidad del ABP, está por debajo del $40 \%$, lo cual se corrobora con los resultados obtenidos en la encuesta y en base a ciertos conceptos explícitos colocados en la fundamentación conceptual de la presente investigación, los mismos que proporcionan suficientes elementos de juicio a la hora del análisis de resultados.

Tabla 4 Actividades de aprendizaje asistido por el docente

\begin{tabular}{|c|c|c|c|c|c|c|c|c|c|c|}
\hline & \multicolumn{2}{|c|}{ Siempre } & \multicolumn{2}{|c|}{ Casi siempre } & \multicolumn{2}{|c|}{ A veces } & \multicolumn{2}{|c|}{ Omisiones } & \multicolumn{2}{|c|}{ Total } \\
\hline & Fr. & Por. & Fr. & Por. & Fr. & Por. & Fr. & Por. & Fr. & Por. \\
\hline $\begin{array}{l}\text { Preg. } 2.1 \mathrm{El} \text { docente } \\
\text { según la clase plantea: } \\
\text { Un problema para re- } \\
\text { solverlo. }\end{array}$ & 23 & $34,8 \%$ & 40 & $60 \%$ & 0 & $0 \%$ & 3 & $4,5 \%$ & 66 & $100 \%$ \\
\hline $\begin{array}{l}\text { Preg. } 2.1 \mathrm{El} \text { docente } \\
\text { según la clase plantea: }\end{array}$ & & & & & & & & & & \\
\hline $\begin{array}{l}\text { Actividades como sumar, } \\
\text { construir, establecer cri- } \\
\text { terios, semejanzas, etc. }\end{array}$ & 47 & $71,2 \%$ & 16 & $24,2 \%$ & 1 & $1,5 \%$ & 2 & $3,0 \%$ & 66 & $100 \%$ \\
\hline $\begin{array}{l}\text { Preg. } 2.2 \text { Los trabajos en } \\
\text { grupo en clase son para: } \\
\text { Realizar exposiciones so- } \\
\text { bre el tema de clase. }\end{array}$ & 33 & $50 \%$ & 32 & $48,4 \%$ & 0 & $0 \%$ & 1 & $1,5 \%$ & 66 & $100 \%$ \\
\hline $\begin{array}{l}\text { Preg. } 2.2 \text { Los trabajos } \\
\text { en grupo en clase son } \\
\text { para: resolver problemas } \\
\text { planteados. }\end{array}$ & 27 & $40,9 \%$ & 35 & $53,0 \%$ & 1 & $1,5 \%$ & 3 & $4,5 \%$ & 66 & $100 \%$ \\
\hline
\end{tabular}

Fuente: Encuesta a estudiantes

Elaborado: Autores 2017

Analizada la tabla 4 de la dimensión dos de la actividad de aprendizaje asistido por el docente (construcción del conocimiento) y de acuerdo a los resultados obtenidos en las encuestas a estudiantes, se evidencia en un 71,2\% que la temática de la clase está encaminada a actividades específicas como sumar, construir, establecer criterios, 
semejanzas, entre otras actividades y que los trabajos en grupo en las horas clase son para realizar exposiciones sobre el tema de la clase, lo que evidencia que no se está trabajando con la metodología del ABP, Esta de acuerdo a la fundamentación conceptual consta de pasos consecutivos como: planteamiento, asesoramiento, dirección del problema, retroalimentación y evaluación final; por el contrario se trabaja con actividades que están encaminadas al logro de la destreza con criterio de desempeño planteada en la planificación, lo cual corrobora que la metodología declarada es el ciclo de aprendizaje y no el ABP.

Tabla 5 Actividades de aprendizaje autónomo

\begin{tabular}{|c|c|c|c|c|c|c|c|c|c|c|}
\hline & \multicolumn{2}{|c|}{ Siempre } & \multicolumn{2}{|c|}{ Casi siempre } & \multicolumn{2}{|c|}{ A veces } & \multicolumn{2}{|c|}{ Omisiones } & \multicolumn{2}{|c|}{ Total } \\
\hline & Fr. & Por. & Fr. & Por. & Fr. & Por. & Fr. & Por. & Fr. & Por. \\
\hline $\begin{array}{l}\text { Preg. } 3.1 \text { En las activi- } \\
\text { dades autónomas el do- } \\
\text { cente propone: La res- } \\
\text { olución de tareas de man- } \\
\text { era individual. }\end{array}$ & 50 & $75,7 \%$ & 15 & $22,7 \%$ & 0 & $0 \%$ & 1 & $1,5 \%$ & 66 & $100 \%$ \\
\hline $\begin{array}{l}\text { Preg. } 3.1 \text { En las activi- } \\
\text { dades autónomas el do- } \\
\text { cente propone: Investiga- } \\
\text { ciones bibliográficas que } \\
\text { dan como resultado un } \\
\text { informe. }\end{array}$ & 10 & $15,1 \%$ & 50 & $75,7 \%$ & 3 & $4,5 \%$ & 3 & $4,5 \%$ & 66 & $100 \%$ \\
\hline $\begin{array}{l}\text { Preg. } 3.1 \text { En las ac- } \\
\text { tividades autónomas el } \\
\text { docente propone: Res- } \\
\text { olución de problemas en } \\
\text { forma grupal. }\end{array}$ & 19 & $28,7 \%$ & 43 & $65,1 \%$ & 1 & $1,5 \%$ & 3 & $4,5 \%$ & 66 & $100 \%$ \\
\hline $\begin{array}{l}\text { Preg. } 3.2 \text { El docente le } \\
\text { proporciona fuera de las } \\
\text { horas de clase tutorías. }\end{array}$ & 7 & $10,6 \%$ & 55 & $83,3 \%$ & 4 & $6,0 \%$ & 0 & $0 \%$ & 66 & $100 \%$ \\
\hline
\end{tabular}

Fuente: Encuesta a estudiantes

Elaborado: Autores 2017

Analizada la tabla 5 de la dimensión de actividades de aprendizaje autónomo y práctico se evidencia en el 75,7\% de los estudiantes manifiesta que los trabajos enviados están orientados a la resolución de ejercicios o tareas de manera individual y en un $83,3 \%$ que el docente no proporciona tutorías u orientaciones fuera de las horas de clases, con lo cual se corrobora que la metodología a emplearse en los procesos de enseñanza-aprendizaje no corresponde al ABP, la misma que de acuerdo a la fundamentación conceptual requiera el trabajo en grupo y la asesoría del docente frente a dudas y dificultades del educando

Tabla 6 Actividades de aprendizaje autónomo

\begin{tabular}{|c|c|c|c|c|c|c|c|c|c|c|}
\hline & \multicolumn{2}{|c|}{$\begin{array}{l}\text { Lee y analiza } \\
\text { el problema }\end{array}$} & \multicolumn{2}{|c|}{$\begin{array}{l}\text { Realiza comentarios } \\
\text { o lluvia de ideas. }\end{array}$} & \multicolumn{2}{|c|}{$\begin{array}{l}\text { Hace una lista } \\
\text { de lo que conoce } \\
\text { y desconoce. }\end{array}$} & \multicolumn{2}{|c|}{$\begin{array}{c}\text { Realiza un } \\
\text { cronograma de } \\
\text { actividades para } \\
\text { realizar el trabajo. }\end{array}$} & \multicolumn{2}{|c|}{$\begin{array}{c}\text { Procede } \\
\text { directamente a } \\
\text { la búsqueda de } \\
\text { información. }\end{array}$} \\
\hline & Fr. & Por. & Fr. & Por. & Fr. & Por. & Fr. & Por. & Fr. & Por. \\
\hline Siempre & 45 & $68,1 \%$ & 16 & $24,2 \%$ & 7 & $10,6 \%$ & 24 & $36,3 \%$ & 25 & $37,8 \%$ \\
\hline A veces & 20 & $30,3 \%$ & 30 & $45,4 \%$ & 39 & $59,0 \%$ & 37 & $56,0 \%$ & 39 & $59,0 \%$ \\
\hline Nunca & 1 & $1,5 \%$ & 20 & $30,3 \%$ & 20 & $30,3 \%$ & 4 & $6,0 \%$ & 2 & $3,0 \%$ \\
\hline Omisiones & 0 & $0 \%$ & 0 & $0 \%$ & 0 & $0 \%$ & 1 & $1,5 \%$ & 0 & $0 \%$ \\
\hline Total & 66 & $100 \%$ & 66 & $100 \%$ & 66 & $100 \%$ & 66 & $100 \%$ & 66 & $100 \%$ \\
\hline
\end{tabular}

Fuente: Encuesta a estudiantes

Elaborado: Autores 2017

Analizada la tabla 6 de la dimensión tres de las actividades de aprendizaje autónomo y práctico se identifica un desconocimiento de los estudiantes para llegar a la resolución de un problema, pues ellos manifiestan en un
$68,1 \%$ que leen y analizan el problema y que posteriormente buscan la resolución al mismo, en porcentajes por debajo del $40 \%$ reconocen que realizan comentarios o lluvias de ideas, elaboran listas de lo que desconocen y realizan cronogramas de trabajo, pasos que de acuerdo a la fundamentación conceptual del ABP deben desarrollarse en iguales proporciones. Además se evidencia que los educandos no siguen la secuencia didáctica en el desarrollo de los ocho pasos planteados para resolver el problema, aspecto que evidencia la no aplicabilidad de ABP en los procesos de enseñanza - aprendizaje.

Tabla 7 Metodología

\begin{tabular}{llllllc}
\hline & \multicolumn{2}{c}{$\mathrm{Si}$} & \multicolumn{2}{c}{ No } & \multicolumn{2}{c}{ Total } \\
\hline & Fr. & Por. & Fr. & Por. & Fr. & Por. \\
$\begin{array}{l}\text { La metodología declara- } \\
\text { da corresponde al (ABP). }\end{array}$ & 0 & $0 \%$ & 84 & $100 \%$ & 84 & $100 \%$ \\
$\begin{array}{l}\text { La metodología declara- } \\
\text { da es el ciclo de aprendi- } \\
\text { zaje. }\end{array}$ & 84 & $100 \%$ & 0 & $0 \%$ & 84 & $100 \%$ \\
\hline
\end{tabular}

Fuente: Ficha de análisis del Plan de Unidad Didáctica

Elaborado: Autores 2017

Analizada la tabla 7 correspondiente a la metodología declarada en el Plan de Unidad Didáctica y de acuerdo a los resultados obtenidos se puede deducir que la metodología a emplearse en los procesos de enseñanza-aprendizaje es el Ciclo del Aprendizaje y no el ABP, con lo cual se descarta que el ABP se aplica en las sesiones de clase tal como lo sostienen en un $60 \%$ los docentes en la encuesta aplicada a esta población. Además se evidencia que se sigue educando con estrategias didácticas tradicionales las mismas que conciben al docente como el dueño del saber $\mathrm{y}$ al educando como un sujeto pasivo y mero receptor de contenidos.

Tabla 8 Actividades de aprendizaje asistido por el docente

\begin{tabular}{lcccccc}
\hline & \multicolumn{2}{c}{$\mathrm{Si}$} & \multicolumn{2}{c}{ No } & \multicolumn{2}{c}{ Total } \\
\hline & Fr. & Por. & Fr. & Por. & Fr. & Por. \\
$\begin{array}{l}\text { Consta el planteamien- } \\
\text { to, asesoramiento y direc- } \\
\text { ción del problema. }\end{array}$ & 0 & $0 \%$ & 84 & $100 \%$ & 84 & $100 \%$ \\
$\begin{array}{l}\text { Desarrolla actividades } \\
\text { para lograr la destreza } \\
\text { con criterio de } \\
\text { desempeño planteada } \\
\text { en la planificación. }\end{array}$ & 84 & $100 \%$ & 0 & $0 \%$ & 84 & $100 \%$ \\
$\begin{array}{l}\text { Los trabajos enviados es- } \\
\text { tán declarados como un } \\
\text { problema. }\end{array}$ & 0 & $0 \%$ & 84 & $100 \%$ & 84 & $100 \%$ \\
$\begin{array}{l}\text { Para el desarrollo del pro- } \\
\text { blema se propone confor- } \\
\text { mar grupos de trabajo. }\end{array}$ & 21 & $25 \%$ & 63 & $75 \%$ & 84 & $100 \%$ \\
\hline
\end{tabular}

Fuente: Ficha de análisis del Plan de Unidad Didáctica Elaborado: Autores 2017

Según la tabla 8 correspondiente al análisis del Plan de Unidad Didáctica, y de acuerdo a los resultados obtenidos se puede establecer en un $100 \%$ que no se aplica la metodología del ABP en la actividades de aprendizaje asistido por 
el docente ni en las actividades autónomas, por el contrario se evidencia en un $100 \%$ el desarrollo de actividades para lograr la destreza con criterio de desempeño planteada en las planificaciones de las diferentes asignaturas, elemento que se corrobora en la encuesta a estudiantes, cuando los mismos reconocen en un 71,2\% que las actividades a desarrollarse en las clases corresponden a establecer ejercicios, semejanzas, entre otras actividades.

En un $75 \%$ se evidencia, el desarrollo de problemas mediante la conformación de grupos, sin embargo los problemas planteados corresponden a enunciados que no evidencian el nivel de complejidad que se requiere para la aplicación de la metodología del ABP; razón por la cual, son resueltos mediante instrucciones enunciadas por el docente, durante la sesión de clase.

Tabla 9 Evaluación

\begin{tabular}{lllllll}
\hline & \multicolumn{2}{c}{$\mathrm{Si}$} & \multicolumn{2}{c}{ No } & \multicolumn{2}{c}{ Total } \\
\hline & Fr. & Por. & Fr. & Por. & Fr. & Por. \\
$\begin{array}{l}\text { Esta declarada la auto- } \\
\text { evaluación del educando. }\end{array}$ & 2 & $2,3 \%$ & 82 & $97,6 \%$ & 84 & $100 \%$ \\
$\begin{array}{l}\text { Se evidencia la coevalua- } \\
\text { ción. }\end{array}$ & 0 & $0 \%$ & 84 & $100 \%$ & 84 & $100 \%$ \\
$\begin{array}{l}\text { La evaluación expresada } \\
\text { es de carácter sumativa } \\
\text { (Solo-Docente). }\end{array}$ & 84 & $100 \%$ & 0 & $0 \%$ & 84 & $100 \%$ \\
\hline
\end{tabular}

Fuente: Ficha de análisis del Plan de Unidad Didáctica Elaborado: Autores 2017

Analizada la tabla 9 correspondiente al Plan de Unidad Didáctica, dimensión cuatro de evaluación y de acuerdo a los resultados obtenidos, se puede deducir que el cien por ciento de la planta docente realiza la evaluación de los aprendizajes, y que es casi nula la autoevaluación pues tan solo en dos de las 84 planificaciones analizadas se evidenció la declaración de autoevaluación, siendo nula la coevaluación, con estos porcentajes se puede reafirmar el desconocimiento de los docentes sobre la metodología del ABP. Además se evidencia que el sistema de evaluación está centrado en el método tradicionalista en donde el docente evalúa conforme su autoridad y no evidenciando el desarrollo de capacidades, competencias y destrezas, aspectos propuestos en la estrategia didáctica del ABP.

\section{Conclusiones}

Realizado el trabajo de investigación El aprendizaje Basado en Problemas como estrategia didáctica en el proceso de enseñanza-aprendizaje, en la Escuela de Educación General Básica José de la Vega, se puede establecer las siguientes conclusiones:

El ABP es una estrategia activa que busca promover el aprender a aprender, desarrollando un aprendizaje significativo en los educandos; es decir, un aprendizaje duradero, el mismo que es recuperado en el momento que se requiere para resolver problemas del diario vivir. Para desarrollar estas competencias en el educando se requiere que esta metodología sea trabajada en grupos pequeños de seis a cuatro estudiantes, los mismos que analizan el problema, reconocen sus necesidades de aprendizaje sobre las cuales buscan la información que les lleve a la resolución del problema. Durante este proceso el educando desarrolla no solo competencias de aprendizaje sino también competencias sociales, las mismas que le permiten hacer y ser tolerante a la crítica, respetar los diferentes puntos de vistas entre otras actitudes. En virtud a lo antes expuesto es posible alcanzar el primer objetivo específico, el mismo que busca conocer los fundamentos conceptuales y teóricos del ABP.

En el nivel de conocimientos que los docentes disponen sobre el ABP, se puede concluir que en cuanto a concepciones generales el nivel de conocimiento está por debajo del $40 \%$, pues los docentes reconocen que el ABP promueve el desarrollo de habilidades de razonamiento, el pensamiento crítico, pero no reconocen como esencial el trabajo en pequeños grupos, técnica que es indispensable en el desarrollo del ABP. Tampoco reconocen la importancia de las tutorías en el desarrollo de esta estrategia, además se evidencia un desconocimiento de parte de los docentes, los cuales no reconocen el proceso didáctico del ABP ni sistema de tutorías que esta metodología requiere, menos aún se evidencia la aplicación integral de los tres componentes de evaluación. Elementos que permiten alcanzar el segundo objetivo específico: nivel de conocimientos de los docentes sobre el ABP.

En cuanto al estado actual de la utilización del ABP en la Escuela José de la Vega se puede manifestar que es nulo, pues las encuestas aplicadas a las estudiantes y el análisis del Plan de Unidad Didáctica evidencian que la metodología empleada es la del ciclo de aprendizaje. Se evidencia que en las actividades de aprendizaje asistido por el docente el trabajo autónomo, y las actividades están encaminadas a tareas individuales que propenden en un alto porcentaje el logro de la destreza con criterio de desempeño planteada en la planificación, tampoco se evidencia la aplicación del proceso de planteamiento, asesoría, dirección, retroalimentación y evaluación del $\mathrm{ABP}$, elementos que permiten alcanzar el tercer objetivo específico: analizar el estado actual de la utilización del ABP.

Concluido el presente trabajo investigativo se puede manifestar que, en el contexto de EGB no se están empleando estrategias metodológicas innovadoras que propendan al desarrollo del conocimiento, por el contrario se sigue trabajando con estrategias convencionales que siguen promoviendo la pasividad del educando en la construcción del conocimiento, reduciendo el desarrollo de las habilidades de razonamiento, de trabajo en equipo, de investigación, comprensión, pero sobre todo limitando su capacidad para aprender a aprender y desarrollar un aprendizaje significativo.

Por tanto, es necesario que se tome conciencia, que la sociedad requiere que las escuelas contribuyan con la formación de los futuros profesionales, los mismos que deben desarrollar no solo un conocimiento teórico sino práctico, para enfrentar y resolver los problemas del diario vivir. Esto 
a su vez requiere que los docentes implementen la estrategia didáctica del ABP, la misma que exige docentes con un solo conocimiento en su sistema de planificación, aplicación y evaluación.

\section{Recomendaciones}

Partiendo del análisis e interpretación de los resultados $\mathrm{y}$ en base a las conclusiones propuestas se establece las siguientes recomendaciones:

A la Escuela "José de la Vega" que indague acerca de mecanismos para implementar de manera paulatina estrategias didácticas que permitan evidenciar la verdadera actividad del educando en la construcción del conocimiento, para que sea el estudiante quien analice el problema planteado, proponga las formas de resolución y por ende sea capaz de plantear la resolución al problema propuesto, lo cual surge de un proceso de investigación, análisis y reflexión, elementos con los cuales se integra la parte teórica y práctica promoviendo así al aprendizaje significativo de los educandos.

Se recomienda a los docentes de EGB, innovar en las sesiones de clases con estrategias didácticas activas, que permitan al educando desarrollar capacidades, actitudes y destrezas para la vida, pero sobre todo se debe tener presente que la estrategia seleccionada debe respetar la realidad de los estudiantes, así como su ritmo y estilo de aprendizaje.

Considerar la posibilidad de implementar la estrategia didáctica del ABP en los procesos de enseñanza - aprendizaje en EGB, para posteriormente medir y evaluar en los educandos su impacto en la construcción del conocimiento.

\section{Referencias Bibliográficas}

Arpí Miró, C., Ávila, P., Baraldés i Capdevila, M., Benito Mundet, H., Gutiérrez del Moral, M. d. J., Orts Alís, M., ... Rostán Sánchez, C. (2012). El ABP: origen, modelos y técnicas afines. Aula de innovación educativa(216), 14-18.

Barrows, H. (1986). Una taxonomía de métodos de aprendizaje basado en problemas. Illinois EE.UU.: Blackwell Publishing Ltd.
Carrasco, J. B. (2004). Una didáctica para hoy. Cómo enseñar mejor. Madrid: Ediciones Rialp.

Chávez, A. (2009). El aprendizaje basado en problemas, como enfoque pedagógico en estudiantes de postgrado de neonatología (Inf. Téc.). Guayaquil.

De innovación educativa, S. (2008). Aprendizaje basado en problemas. Guías rápidas sobre nuevas metodologías (Inf. Téc.). Madrid: Servicio de innovación educativa de la Universidad Politécnica de Madrid.

Escribano, A., y Del Valle,. (2008). El aprendizaje basadoen problemas. Una propuesta metodológica en Educación Superior. Madrid: Editorial Narcea, S.A.

Monterrey, D. (2008). Las estrategias y técnicas didácticas en el rediseño: el aprendizaje basado en problemas como técnica didáctica. México.

Morales Bueno, P., y Landa Fitzgerald, V. (2004). Aprendizaje basado en problemas. Theoria, 13, 145-157.

Palacios, R. (2006). Investigación cualitativa y cuantitativa - Diferencias y limitaciones. , s.p.

Palta, N. (2016). El aprendizaje basado en problemas como estrategia didáctica para la carrera de sicología clínica (Inf. Téc.). Guayaquil: Universidad Católica Santiago de Guayaquil.

Poot-Delgado, C. A. (2013). Retos del aprendizaje basado en problemas. Enseñanza e investigación en psicología, 18(2), 307-314.

Recibido: 18 de junio de 2018

Aceptado: 29 de junio de 2018

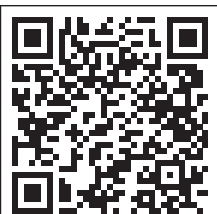


